\title{
Epidemiology of Patellar Tendinopathy in Elite Male Soccer Players
}

\author{
Martin Hägglund, Johannes Zwerver and Jan Ekstrand
}

\section{Linköping University Post Print}

\section{Tweet}

N.B.: When citing this work, cite the original article.

Original Publication:

Martin Hägglund, Johannes Zwerver and Jan Ekstrand, Epidemiology of Patellar Tendinopathy in Elite Male Soccer Players, 2011, American Journal of Sports Medicine, (39), 9, 1906-1911.

http://dx.doi.org/10.1177/0363546511408877

Copyright: SAGE Publications (UK and US): No SAGE Choice http://www.uk.sagepub.com/home.nav

Postprint available at: Linköping University Electronic Press

http://urn.kb.se/resolve?urn=urn:nbn:se:liu:diva-70735 


\section{Epidemiology of patellar tendinopathy in elite male soccer players}

3

4

5 Martin Hägglund, ${ }^{*} \mathrm{PT}, \mathrm{PhD}$, Johannes Zwerver, $\uparrow \mathrm{MD}, \mathrm{PhD}$, Jan Ekstrand, $\uparrow \mathrm{MD}, \mathrm{PhD}$

6

7

$8 *$ Department of Medical and Health Sciences, Division of Physiotherapy, Linköping

9 University, Sweden

10

$11 \uparrow$ Center for Sports Medicine, University Center for Sport, Exercise and Health, University

12 Medical Center Groningen, University of Groningen, The Netherlands

13

14 † Department of Medical and Health Sciences, Division of Health and Society, Linköping

15 University, Sweden

16

17 Corresponding author

18 Martin Hägglund

19 Department of Medical and Health Sciences, Division of Physiotherapy, Linköping

20 University, SE-581 83 Linköping, Sweden 


\section{ACKNOWLEDGEMENTS}

2 The participating clubs with all their players, coaching staff and medical staff are gratefully

3 acknowledged. This study was supported by grants from the Union of European Football

4 Associations and the Swedish Centre for Research in Sports. 
2 Title

3 Epidemiology of patellar tendinopathy in elite male soccer players

4

5

6

7

8

9 


\section{Abstract}

2 Background: Patellar tendinopathy is common among athletes in jumping sports and in

3 sports with prolonged repetitive stress of the knee extensor apparatus. The epidemiology in

4 soccer is not well described.

5 Purpose: To study the epidemiology of patellar tendinopathy in elite male soccer players

6 and evaluate potential risk factors.

7 Study Design: Prospective cohort study

8 Methods: 51 European elite soccer clubs (2,229 players) from three different cohorts,

9 Swedish First League cohort (SWE) and UEFA Champions League cohort (UCL), both

10 playing on natural grass, and Artifical Turf cohort (ART) playing on third generation artificial

11 turfs, were followed between 2001 and 2009. Individual player exposure in training and

12 matches and time-loss injuries were recorded.

13 Results: In total, 137 patellar tendinopathies were recorded, comprising $1.5 \%$ of all injuries

14 and corresponding to an incidence of 0.12 injuries/1000 hours. Each season, $2.4 \%$ of players

15 were affected, with most injuries (61\%) resulting in absence up to one week or less. Twenty

16 per-cent of tendinopathies were recurrent complaints. No significant difference in season

17 prevalence (OR $\mathbf{0 . 9 3 , 9 5 \% ~ C I ~} \mathbf{0 . 6 0 - 1 . 4 4 , ~} \mathrm{p}=\mathbf{0 . 7 4}$ ) or incidence (RR 1.20, 95\% CI 0.82-1.75,

$18 \mathbf{p}=\mathbf{0 . 3 6}$ ) was observed between teams playing on artificial turf and natural grass respectively.

19 Multivariate logistic regression showed that a high total exposure hours (OR 1.02 per 10

20 hour increase; $95 \%$ CI 1.00-1.04, p=0.033) was a significant risk factor for patellar

21 tendinopathy, and increased body mass was borderline significant (OR 1.15 per 5 kg

22 increase; 95\% CI 1.00-1.33, p=0.055). In addition, two acute partial tendon ruptures

23 were recorded, but no total rupture.

24 Conclusions: Although mainly mild in nature, patellar tendinopathy is a fairly common

25 condition in elite soccer and the recurrence rate is high. Exposure to artificial turf did not 
1 increase the prevalence or incidence of injury. High total amount of exposure was identified

2 as a risk factor for patellar tendinopathy.

3

4 Key terms: Patellar tendinopathy - tendinosis - jumper's knee - football - artificial turf

7 What is known about the subject: Patellar tendinopathy (PT) is common in jumping sports 8 and in sports with prolonged repetitive stress of the knee extensor apparatus, such as soccer.

9 The epidemiology of PT in soccer is not well described, and the existing studies are limited by

10 small samples. The evidence is limited regarding risk factors for PT in sports, with many

11 conflicting results in the literature. In soccer, only few potential risk factors have been

12 evaluated and data are available mainly from cross-sectional studies.

13

14 What this study adds to the existing knowledge: This was the first well sized prospective

15 cohort study describing the epidemiology and risk factors for PT in soccer. The results concur

16 with previous studies from other sports showing that a high overall exposure is a risk factor

17 for PT, and increased body mass was borderline significant. In addition, incidence of PT was

18 high during the pre-season when training load is increased, suggesting a link between tendon

19 load and time-loss due to PT in soccer players. Exposure to soccer play on artificial turf was

20 not associated with an increased risk of PT. 


\section{INTRODUCTION}

2 Patellar tendinopathy (PT) is a common condition among athletes characterized by gradually

3 progressive activity-related anterior knee pain and focal patellar tenderness (29). Symptoms

4 are often serious leading to recurrent or long-standing impairment of athletic performance

5 (20). Currently, there is no consensus on what is the most appropriate treatment of PT and

6 treatment results are often disappointing $(3,26)$. Patellar tendinopathy can thus have a major

7 impact on the career of many athletes and for some it is even the reason to end their career

8 prematurely (18).

9

10 Historically, it has often been referred to as jumper's knee because the prevalence is

11 especially high in jumping sports. Lian et al. reported a current prevalence of $45 \%$ in elite

12 volleyball and $32 \%$ in elite basketball players (20). However, also in other sports like soccer

13 a prolonged repetitive stress of the knee extensor apparatus can lead to this overuse

14 tendinopathy of the patellar tendon $(17,20)$. Its etiology has not been elucidated completely so

15 far but seems to be multifactorial. Evidence is limited regarding risk factors for PT with

16 conflicting results in the literature. Some of the suggested intrinsic risk factors are male

17 gender (20), high stature (20), high body mass $(4,20,22,23)$, and reduced ankle dorsiflexion

18 (24) while extrinsic factors include increased training frequency $(4,7,11)$, high frequency of

19 weight and jump training $(20,22)$ and, in volleyball, playing on hard surfaces $(1,7)$.

21 Soccer, one of the most popular sports with about 260 million players worldwide, causes a

22 large amount of acute and chronic injuries in both male and female players of every age and at

23 all playing levels (16). Up to now most attention in soccer related medical research has been

24 paid to the epidemiology, prevention and clinical management of acute injuries such as knee,

25 ankle and hamstring injuries, conditions which give rise to significant morbidity in soccer 
1 players and that can also result in high medical costs and financial burden for professional

2 soccer teams (6). Even though PT might also negatively influence the athletic performance

3 and career of soccer players, surprisingly few research papers have been published about

4 patellar tendon injuries involving soccer players $(8,9,19,20)$. Furthermore, the few studies that

5 have been published are limited by small samples.

6

7 Therefore, the aim of this study was to describe the epidemiology of PT in a large population 8 of elite male soccer players and to identify potential risk factors.

9 
MATERIALS AND METHODS

2

\section{Participants and study cohorts}

4 Data was derived from three prospective cohort studies of European elite men's soccer carried

5 out between 2001 and 2009. The study cohorts comprised in total 51 teams (2,299 players)

6 followed over a varying number of seasons; the UEFA (Union of European Football

7 Associations) Champions League cohort (UCL) (6,30) included 24 European professional 8 teams from 10 countries followed between July 2001 and June 2009 (mean 4.3 seasons or

945.3 months participation per team) and consisted of 1210 players; the Swedish First League cohort $(S W E)(13,14)$ comprised 15 Swedish top division teams followed over three seasons

11 (January to November) 2001, 2002 and 2005 (mean 2.3 seasons or 23.3 months/team) with

12508 players; and the Artifical Turf cohort (ART) (5) included 15 European elite teams (top two 13 domestic divisions) from eight countries followed between February 2003 and December 142009 (mean 3.3 seasons or 30.3 months/team) with 661 players. All contracted players in the 15 first teams were invited to participate in the study. Players who left the team during the 16 season, e.g. due to transfer, were included during their time in the team. Anthropometrics and 17 exposure data for the three cohorts are shown in Table 1.

Teams were followed during the full soccer season, including pre-season and competitive season. Teams in the UCL and SWE cohorts trained mainly on natural grass and played their

21 home matches on natural grass. Teams in the ART cohort all played on third generation 22 artificial turfs at their home grounds and also trained mainly on artificial turf, while away matches were played mainly on natural grass. Three teams were initially included in the SWE cohort (seasons 2001 and 2002) and then entered the ART cohort due to a change from natural grass to artificial turf playing surface. Exposure and injury data from these three 
teams were included in the SWE cohort during the 2001 and 2002 seasons whilst playing

2 on natural grass, and then included in the ART cohort from season 2003 after the

3 change of playing surface.

4

Table 1 near here

6

$7 \quad$ Data collection and study definitions

8 The study design followed the definitions and data collection procedures outlined in the

9 consensus document (10) and by UEFA (12) for studies of soccer injuries. The validation of

10 the injury and exposure reporting system and definitions has been described previously (12).

11 To ensure high reliability of data registration all teams were provided with a study manual

12 describing the definitions used and procedures to record data, including explanatory examples. In addition, all reports were checked each month by the study group and feedback sent to the teams in order to correct any missing or unclear data. The definitions applied in the study are shown in Table 2.

16

Player baseline data was collected once yearly, at the start of each season. Individual player participation in training and matches (minutes of exposure) was registered by the club contact person on a standard exposure form sent to the study group on a monthly basis. This included exposures with the first and second team, as well as any national team exposure, for all

21 players. The training content was not recorded. The team medical staffs recorded all time-

22 loss injuries on a standard injury form that was sent to the study group each month. The injury form provided information about the diagnosis, nature and circumstances of injury occurence.

24 Injuries were categorised under four degrees of severity based on the number of days' 25 absence. All injuries were followed until the final day of rehabilitation. For the present 
1 study we included all recorded patellar tendon injuries, comprising both gradual onset

2 patellar tendinopathies and acute onset patellar tendon partial ruptures. The diagnosis

3 was based on the clinical examination by the team medical staff, and no specific diagnostic

4 criteria were sent out in advance.

Table 2 near here

7

\section{Risk factor evaluation}

We collected information about potential intrinsic risk factors for all players when entering the study; age, stature, body mass and playing position (goalkeeper, defender, midfielder, forward). The extrinsic risk factors evaluated in the study were total exposure hours (hours of soccer training and match play), training/match exposure ratio (hours of training/hours of match play), team home surface (natural grass or artificial turf), and seasonal distribution (autumn to spring or spring to autumn season).

\section{Statistical analyses}

ANOVA was used for group comparisons of continuous normally distributed data, the MannWhitney U-test or Wilcoxon signed rank test for continuous non-normally distributed data and the $\chi^{2}$ test for categorical data. Injury incidence was calculated as the number of injuries per 1,000 player hours, and compared between groups with rate ratios (RR) with $95 \%$ confidence intervals $(95 \% \mathrm{CI})$, and significance tested using z-statistics. Season injury prevalence was calculated as the number of injured players during a season/total number of players in that season $\mathrm{x}$ 100, and was compared between groups with odds ratios (OR) and corresponding $95 \% \mathrm{CI}$, and significance tested with z-statistics. The risk factor analysis was made with the player as unit for analysis, and odds ratios (OR) with $95 \% \mathrm{CI}$ are presented 
1 for all variables based on univariate logistic regression. For the multivariate analysis we

2 included all variables in a backward stepwise logistic regression, and variables with $\mathrm{p}<0.20$

3 were included in the final model. The significance level was set at $\mathrm{p}<0.05$ for all analyses.

4

5 The study design underwent an ethical review and was approved by the UEFA Football

6 Development Division and the UEFA Medical Committee.

7

8 


\section{RESULTS}

2 In total, 139 patellar tendon injuries were registered; 137 of which were gradual onset tendinopathies (PT) and two were acute onset partial tendon tears. The two acute partial

4 tears affected one player in the UCL and ART cohorts respectively, and due to their

5 different aetiology (acute onset) these two injuries are not included in the following

6 results section. No total patellar tendon rupture was recorded.

$8 \quad$ Nature and severity of patellar tendinopathies

9 Four injuries affected the distal patellar tendon, and the remaining 133 were proximal. Fiftyfive injuries $(40 \%)$ affected the dominant leg (preferred shooting leg), 66 injuries (48\%) the

11 non-dominant leg ( $\mathrm{p}=\mathbf{0 . 3 6}$ vs dominant leg), 4 injuries ( $3 \%$ ) affected both knees, and for 12 12 injuries (9\%) the leg dominance was unknown.

14 PT constituted $1.5 \%$ of the total number of injuries registered in the three cohorts, and caused $1.4 \%$ of the total injury absence in the clubs (Table 3 ). The majority of injuries resulted in 16 absence less than one week (61\%), while $10 \%$ were severe, causing absence more than 4 17 weeks from training and match play. Two of the severe injuries were surgically treated. The median absence due to PT was 5 days, with $75 \%$ of players returning within 12 days. One in five injuries was a recurrent complaint, with no difference in absence between recurrent and index injuries (median 5 days for both, $\mathrm{p}=\mathbf{0 . 5 8}$ ). No inter-cohort differences

21 were observed for recurrence rates $(p=0.29)$, injury severity $(p=0.90)$ or median days absence 22 per injury $(\mathrm{p}=\mathbf{0 . 7 1})$.

Table 3 near here 


\section{Season prevalence and incidence}

2 Each season, $2.4 \%$ of players (season prevalence) missed training or match playing time due to PT, with an incidence of 0.12 injuries/1,000 hours of total exposure (Table 3). No

4 significant difference in season prevalence or incidence of PT was observed between teams

5 playing on artificial turf and those playing on natural grass; prevalence $2.25 \%$ vs $2.42 \%$ (OR

$6 \quad \mathbf{0 . 9 3}, 95 \%$ CI 0.60-1.44, $\mathrm{p}=\mathbf{0 . 7 4}$ ); incidence $\mathbf{0 . 1 3}$ vs $0.11 / 1,000$ hours (RR $\mathbf{1 . 2 0 , 9 5 \% ~ C I ~}$

$7 \quad 0.82-1.75, \mathrm{p}=0.36)$.

9 The distribution of PT over the season is shown in Figure 1. For teams following an springto-autumn season the incidence of PT was increased during the pre-season (January to March)

11 compared to the competitive season (April to November) (0.18 vs 0.10/1000 hours, RR 1.86; 95\% CI 1.08-3.20, $\mathrm{p}=0.026)$. The incidence for teams following an autumn-to-spring season peaked in July, October and April.

Figure 1 near here

\section{Risk factors for PT}

18 Results of the univariate logistic regression showed that total exposure hours during a season was the only variable significantly associated with PT (Table 4). In the backward stepwise multivariate logistic regression three variables were included in the final model; total exposure hours (OR 1.02 per 10 hour increase; $95 \%$ CI 1.00-1.04, p=0.033), body weight 


\section{1}

2

3 The main finding of this study was that during the season $2.4 \%$ of all elite soccer players

4 missed training or match playing time due to PT. The absence because of PT was rather short,

5 in the majority of cases less than one week, but the recurrence rate was high. There was no

6 difference in incidence or prevalence of PT from playing on artificial turf or natural grass.

7 High total amount of exposure was identified as a risk factor for PT. The strength of the study

8 is the prospective design and that a large population of elite athletes was followed using the

9 same methodology that complied with international guidelines $(10,12)$.

\section{Prevalence and incidence of PT}

12 Few studies have reported on the epidemiology of PT in soccer. Lian et al. described a high, 13

$23 \%$, overall prevalence (present and previous symptoms) of PT in Norwegian elite male soccer players, with a point prevalence of $13 \%(20)$. However, these data are difficult to compare with our season prevalence since non time-loss injuries were included. Studies from Danish elite male soccer show that $7 \%$ of players (3.1 to $7 \%$ of patellar tendons) developed symptomatic PT during a season $(8,9)$. Interestingly, as many as $6 \%$ of players $(3 \%$ of patellar tendons) reported time loss due to PT (9), indicating that over the season a majority of players with PT developed symptoms severe enough to limit performance and leading to missed training or match exposure. The season prevalence found in the present study (2.3-2.6 $\%$ ) was less than half of that reported previously, but it is not known whether this reflects a true difference in injury prevalence or differences in study methodology, reporting thresholds or varying treatment regimes (i.e. to let players with symptoms carry on playing or take them out of training). We found no difference in incidence or prevalence of PT between the three different cohorts in our study, representing the highest professional European level (UCL), 
1 and high European elite level (ART, SWE). Noteworthy is that only two acute onset partial

2 ruptures were identified in this study, and no complete rupture, indicating that these are

3 rare conditions in elite soccer. Tendon rupture is considered the final stage of PT (21)

4 and one could speculate that by early identification and treatment of the condition, and

5 by a reduction of tendon load (e.g. alternative training programmes) during

6 symptomatic periods, tendon ruptures were avoided in these elite soccer players.

8 High recurrence rate

9 The high recurrence rate found in this study (12-27\%) reflects the chronic and recurrent

10 character of this troublesome condition, and corresponds with previous studies of male elite

11 soccer (21\%) (9). After a short period of rest, load reduction and treatment athletes become

12 asymptomatic and may restart competing until another period of symptoms occurs. From studies in volleyball players it is clear that pain and ultrasonographic abnormalities can vary over the season (25), being indicative of players having recurrent complaints. There is, unfortunately, little evidence on how to prevent these recurrent episodes of PT in athletes.

16 Eccentric training, a cornerstone in the conservative treatment of chronic PT $(26,28)$, has not shown similarly good results for prevention (9). However, correction of intrinsic and extrinsic risk factors should be an important part in prevention. For instance, an adapted training programme may be advised to athletes with a previous history of PT during parts of the season involving increased load, e.g. during the pre-season preparation or during intense parts

21 of the competitive season, in order to prevent recurrent complaints.

\section{Risk factors for PT}

24 In our group of elite male soccer players, a high total amount of exposure was associated with 25 an increased risk of PT. This is in line with previous cross-sectional studies from volleyball 
1 (7), basketball (11) and various sports (4) where an association between training load/hours of

2 sports participation and prevalence of PT was observed. The finding is probably indicative of

3 the condition's overuse nature with prolonged repetitive stress of the patellar tendon. For

4 northern European clubs, following a spring to autumn season, an increase in incidence of PT

5 was observed during the pre-season period when training load is high, thus possibly

6 supporting an association between tendon load and development of symptomatic PT. In

7 contrast, Lian et al. reported no difference in overall training amount, amount of sport-specific

8 training, weight training, jump training or other types of training between soccer players with

9 current or previous complaints of PT and those without in their cross-sectional study (20).

11 Of the potential anthropometric risk factors evaluated in the present study, only increased body mass was borderline significant. Increased body mass has been identified as a risk factor in previous studies in volleyball players $(4,20,22,23)$ and may be due to increased

14 forces acting on the patellar tendon with an increased body mass. Stature was not associated 15 with development of PT in our study, which is in agreement with most studies $(4,11,22,23)$, 16 while Lian et al. reported, from their cross-sectional study, that increased stature was 17 associated with the risk of PT in soccer players (20). Similar to previous studies $(4,11,20,22)$ age was not associated with the risk of PT in our group of elite soccer players. Furthermore, we found no association between leg dominance or playing position and development of PT.

21 We found no influence on injury rates from playing surface, whether mainly played on 22 artificial turf or natural grass. Previous reports from the same cohort of elite clubs playing on artificial turf showed that the incidence of acute injuries is also comparable between surfaces

24 (5). Ferretti et al. showed, in a study of volleyball players, that the prevalence of PT increased with a harder playing surface (7) and another study (1) reported a lower prevalence of PT in 
1 beach volleyball compared to previous studies from indoor volleyball, indicating that ground

2 hardness may be correlated with PT. The elite soccer clubs in the ART cohort in our study all

3 played on home grounds with third generation artificial turfs, developed to resemble natural

4 grass and using sand and rubber granules, and sometimes a shock absorbing rubber pad, to

5 reduce ground hardness. Considering that the incidence of PT among northern European

6 teams in our study was high during pre-season, a period with poorer weather and cold

7 temperatures, a possible association between climate conditions, ground hardness and injury

8 rates in soccer should be evaluated further.

9

Methodological considerations

11 First, a limitation of the study is related to the registration of injuries. No specific set

diagnostic criteria for a patellar tendon injury were used. We followed the football consensus proposal for general definitions, and all patellar tendon injuries leading to time loss from training or match play were reported by the team medical staff. Furthermore, we did not collect specific data on the severity of patellar tendinopathies (e.g. VISA-P score (27)) other than time loss due to the injury. No imaging data were obtained from the injured football players either. Therefore, only symptomatic PT and acute onset patellar tendon partial ruptures, diagnosed on the clinical experience of the team medical staff, were included. On the other hand, the correlation between imaging (MRI or ultrasound) and clinical findings is often low $(9,25)$ and clinical examination will identify players with symptomatic patellar tendon injury during soccer training and/or match play. Finally, with the current time loss definition used it should be recognised that the injuries captured in our surveillance study probably represents the tip of the iceberg and our data might underestimate the magnitude and impact of this chronic overuse injury (2). It is well known that many athletes with PT may keep on participating in training and matches despite having some pain. However, the 
definition was chosen for the general injury surveillance study to capture injuries with

2 substantial effect on players' health and performance as well as on the performance of

3 the team (12).

4

5 Second, another limitation refers to the registration of training exposures. We only registered

6 collective team training, and not any other individual training (e.g. strength and conditioning

7 training) that players performed as a part of their daily routines in the club, or outside of the

8 club. Therefore, the total training load of players is not known. In order to control for such

9 factors it has been recommended that individual activities should be recorded also in team

10 sports like soccer (15). Furthermore, the intensity and type of training performed was not

11 registered, and this is another limiting factor since it has been shown that high volumes of

12 jump training and eccentric load may increase the risk of PT (20,22). However, even though

13 this may slightly interfere with inter-sport comparisons, it is unlikely that the type of training

14 differs substantially between the different cohorts of elite male football players included in

15 this study and that this would affect our evaluated risk factors (e.g. comparison between

16 teams playing on artificial turf and natural grass).

17

18 CONCLUSION

19 Patellar tendinopathy is a rather common condition in elite soccer players, with a high

20 recurrence rate. High overall exposure during a season was a risk factor for developing PT.

21 Furthermore, an increased incidence of PT was observed during the pre-season period when

22 training load is high. These findings indicate a link between patellar tendon load and injury

23 occurrence, even though results should be confirmed in future studies. More high-quality

24 studies on risk factors for PT in professional as well as in female, amateur and youth soccer

25 are needed to generate preventive strategies. 


\section{REFERENCES}

2 1. Bahr R, Reeser JC. Injuries among world-class professional beach volleyball players. The

3 Fédération Internationale de Volleyball beach volleyball injury study. Am J Sports Med

$4 \quad 2003 ; 31: 119-25$.

2. Bahr R. No injuries, but plenty of pain? On the methodology for recording overuse symptoms in sports. Br J Sports Med 2009;43:966-72.

3. Cook JL, Khan KM. What is the most appropriate treatment for patellar tendinopathy? $\mathrm{Br}$ J Sports Med 2001;35:291-4.

4. Crossley KM, Thancanamootoo K, Metcalf BR, Cook JL, Purdam CR, Warden SJ. Clinical features of patellar tendinopathy and their implications for rehabilitation. $J$ Orthop Res 2007;25:1164-75.

5. Ekstrand J, Hägglund M, Fuller CW. Comparison of injuries sustained on artificial turf and grass by male and female elite football players. Scand J Med Sci Sports 2010, [Epub ahead of print] PMID: 20456680

6. Ekstrand J, Hägglund M, Waldén M. Injury incidence and injury pattern in professional football - the UEFA injury study. Br J Sports Med 2010, [Epub ahead of print] PMID:

7. Ferretti A, Puddu G, Mariani PP, et al. Jumper's knee: an epidemiological study of volleyball players. Physician Sportsmed 1984;12:97-106.

8. Fredberg U, Bolvig L. Significance of ultrasonographically detected asymptomatic tendinosis in the patellar and Achilles tendons of elite soccer players. A longitudinal

9. Fredberg U, Bolvig L, Andersen NT. Prophylactic training in asymptomatic soccer study. Am J Sports Med 2002;30:488-91. players with ultrasonographic abnormalities in achilles and patellar tendons. The Danish super league study. Am J Sports Med 2008;36:451-60. 
1 10. Fuller CW, Ekstrand J, Junge A, et al. Consensus statement on injury definitions and data

2 collection procedures in studies of football (soccer) injuries. Br J Sports Med 2006;40-

$3 \quad 3: 193-201$.

4 11. Gaida JE, Cook JL, Bass SL, Austen S, Kiss ZS. Are unilateral and bilateral patellar tendinopathy distinguished by differences in anthropometry, body composition, or muscle strength in elite female basketball players? Br J Sports Med 2004;38:581-5.

12. Hägglund M, Waldén M, Bahr R, Ekstrand J. Methods for epidemiological study of injuries to professional football players: developing the UEFA model. Br J Sports Med 2005;39:340-6.

13. Hägglund M, Waldén M, Ekstrand J. Injuries among male and female elite football players. Scand J Med Sci Sports 2009,19: 819-27.

14. Hägglund M, Waldén M, Ekstrand J. Previous injury as a risk factor for injury in elite

19. Kraemer R, Knobloch K. A soccer-specific balance training program for hamstring

17. Kannus P. Etiology and patophysiology of chronic tendon disorders in sports. Scand $J$

15. Hägglund M, Waldén M, Til L, Pruna R. The importance of epidemiological research in sports medicine. APUNTS Medicina de l'Esport 2010 DOI:10.1016/j.apunts.2010.02.006

16. Junge A, Dvorak J. Soccer injuries. A review on incidence and prevention. Sports Med 2004;34:929-38. muscle and patellar and Achilles tendon injuries. An intervention study in premier league female soccer. Am J Sports Med 2009;37:1384-93. 
1 20. Lian $\varnothing$, Engebretsen L, Bahr R. Prevalence of jumper's knee among elite athletes from different sports. A cross-sectional study. Am J Sports Med 2005;33:559-67.

21. Lian Ø, Holen KJ, Engebretsen L, Bahr R. Relationship between symptoms of jumper's knee and the ultrasound characteristics of the patellar tendon among high level male volleyball players. Scand J Med Sci Sports 1996;6:291-6.

22. Lian $\emptyset$, Refsnes PE, Engebretsen L, Bahr R. Performance characteristics of volleyball players with patellar tendinopathy. Am J Sports Med 2003;31:408-13.

23. Malliaras P, Cook JL, Kent P. Anthropometric risk factors for patellar tendon injury among volleyball players. Br J Sports Med 2007;41:259-63.

24. Malliaras P, Cook JL, Kent P. Reduced ankle dorsiflexion range may increase the risk for patellar tendon injury among volleyball players. J Sci Med Sport 2006;9:304-9.

25. Malliaras P, Cook J, Ptasznik R, Thomas S. Prospective study of change in patellar tendon abnormality on imaging and pain over a volleyball season. Br J Sports Med 2006;40:27274.

26. Peers K, Lysens R. Patellar tendinopathy in athletes. Current diagnostic and therapeutic recommendations. Sports Med 2005;35:71-87.

27. Visentini PJ, Khan KM, Cook JL, Kiss ZS, Harcourt PR, Wark JD. The VISA score: an index of severity of symptoms in patients with jumper's knee (patellar tendinosis). Victorian Institute of Sport Tendon Study Group. J Sci Med Sport 1998;1:22-8.

28. Visnes H, Bahr R. The evolution of eccentric training as treatment for patellar tendinopathy (jumper's knee): a critical review of exercise programmes. Br J Sports Med 2007;41:217-23.

29. Warden SJ, Brukner P. Patellar tendinopathy. Clin Sports Med 2003;22:743-59 
1 30. Waldén M, Hägglund M, Ekstrand J. UEFA Champions League study: a prospective study

2 of injuries in professional football during the 2001-2002 season. Br J Sports Med $3 \quad 2005 ; 39: 542-6$.

4 
1 31. Table 1. Player anthropometrics and exposures in three cohorts of elite male soccer players.

\begin{tabular}{|c|c|c|c|}
\hline & $\mathrm{UCL}^{\top}$ & SWE $^{1}$ & $\mathrm{ART}^{1}$ \\
\hline No. of teams (team seasons ${ }^{2}$ ) & $24(104)$ & $15(35)$ & $15(49)$ \\
\hline No. of players (player seasons ${ }^{2}$ ) & $1210(2686)$ & $508(774)$ & $661(1198)$ \\
\hline \multicolumn{4}{|l|}{ Player anthropometrics ${ }^{3}$} \\
\hline Age (years) & $25.7 \pm 4.5$ & $24.8 \pm 4.7$ & $25.0 \pm 4.8$ \\
\hline Height $(\mathrm{cm})$ & $181.7 \pm 6.4$ & $182.5 \pm 5.7$ & $181.8 \pm 6.2$ \\
\hline Weight (kg) & $78.0 \pm 7.0$ & $79.0 \pm 6.1$ & $78.1 \pm 6.9$ \\
\hline \multicolumn{4}{|l|}{ Player exposures (per season) ${ }^{3}$} \\
\hline No. of training sessions & $161 \pm 55$ & $184 \pm 50$ & $144 \pm 69$ \\
\hline Training hours & $210 \pm 73$ & $262 \pm 71$ & $193 \pm 89$ \\
\hline No. of matches & $33 \pm 17$ & $30 \pm 12$ & $26 \pm 15$ \\
\hline Match hours & $41 \pm 24$ & $38 \pm 17$ & $32 \pm 20$ \\
\hline No. of total activities & $195 \pm 67$ & $214 \pm 59$ & $170 \pm 80$ \\
\hline Total training + match hours & $250 \pm 87$ & $300 \pm 83$ & $225 \pm 104$ \\
\hline
\end{tabular}

2 I UEFA Champions League (UCL), Swedish First League (SWE), Artificial Turf (ART)

$3{ }^{2}$ One team or player participating in one season equals one team and one player season respectively

$4 \quad{ }^{3}$ Values are mean \pm standard deviation

5 
Table 2. Study definitions.

\begin{tabular}{ll}
\hline Training session & Team training that involved physical activity under the supervision of the coaching staff. \\
Match & Competitive or friendly match against another team. \\
Injury & Injury resulting from playing football and leading to a player being unable to fully participate \\
& in future training or match play (i.e. time-loss injury). \\
Patellar tendon & Overuse (tendinopathy) or traumatic distraction injury (partial or total tear) located to the \\
injury & patellar tendon leading to a player being unable to fully participate in training or match play. \\
Rehabilitation & A player was considered injured until team medical staff allowed full participation in \\
& training and availability for match selection. \\
Re-injury & Injury of the same type and at the same site as an index injury occurring no more than two \\
months after a player's return to full participation from the index injury. \\
Minimal injury & Injury causing absence of $1-3$ days from training and match play. \\
Mild injury & Injury causing absence of $4-7$ days from training and match play. \\
Moderate injury & Injury causing absence of $8-28$ days from training and match play. \\
Severe injury & Injury causing absence of over 28 days from training and match play. \\
Traumatic injury & Injury with sudden onset and known cause. \\
Overuse injury & Injury with insidious onset and no known trauma. \\
Season prevalence & Number of injured players in a season/total number of players in the same season $\times 100$ \\
Injury incidence & Number of injuries per 1,000 player hours $[(\Sigma$ injuries/ $\Sigma$ exposure hours $) \times 1,000]$.
\end{tabular}


1 Table 3. Nature, prevalence and incidence of patellar tendinopathy ${ }^{1}$.

\begin{tabular}{lllll}
\hline & Total & $\mathrm{UCL}^{2}$ & $\mathrm{SWE}^{2}$ & $\mathrm{ART}^{2}$ \\
\hline No of patellar tendinopathies & 137 & 76 & 25 & 36 \\
Percentage of total no of injuries & $1.48 \%$ & $1.43 \%$ & $1.43 \%$ & $1.62 \%$ \\
Injury severity & & & & $14(39 \%)$ \\
$\quad$ Minimal (1-3 days) & $57(42 \%)$ & $31(41 \%)$ & $12(48 \%)$ & $8(22 \%)$ \\
$\quad$ Mild (4-7 days) & $27(20 \%)$ & $16(21 \%)$ & $3(12 \%)$ & $9(25 \%)$ \\
$\quad$ Moderate (8-28 days) & $39(28 \%)$ & $22(29 \%)$ & $8(32 \%)$ & $5(14 \%)$ \\
$\quad$ Severe (>28 days) & $14(10 \%)$ & $7(9 \%)$ & $2(8 \%)$ & $6(2 ; 15)$ \\
Days absence/injury & $5(2 ; 12)$ & $4.5(3 ; 12)$ & $4(2 ; 9.5)$ & 0.53 \\
Days absence/player/season & 0.44 & 0.43 & 0.31 & $1.96 \%$ \\
Percentage of total injury absence & $1.29 \%$ & $1.17 \%$ & $0.92 \%$ & $2.33 \%$ \\
Season prevalence & $2.38 \%$ & $2.37 \%$ & $2.58 \%$ & $0.13(0.10-0.19)$ \\
Injury incidence & $0.12(0.10-0.14)$ & $0.11(0.09-0.14)$ & $0.11(0.07-0.16)$ & 2.37 \\
Injury burden & 1.74 & 1.72 & 1.04 & $3(12 \%)$ \\
Re-injuries $^{5}$ & $27(20 \%)$ & $14(18 \%)$ & $10(28 \%)$
\end{tabular}

2 Two acute onset patellar tendon partial ruptures (one in the UCL and ART cohorts respectively) are not

3 included in the table

$4 \quad{ }^{2}$ UEFA Champions League (UCL), Swedish First League (SWE), Artificial Turf (ART)

$5 \quad{ }^{3}$ Median $(25 \% ; 75 \%$ quartiles)

$6{ }^{4}$ Season prevalence expressed as number of players with an injury in a season/total no of players that season $\times$

$7 \quad 100$

$8{ }^{5}$ Injury incidence expressed as number of injuries/1000 hours of total exposure (95\% CI)

$9{ }^{6}$ Injury burden expressed as number of days absence/1000 hours of total exposure 
1 Table 4. Results of univariate logistic regression for potential risk factors for patellar tendinopathy.

\begin{tabular}{llll}
\hline Risk factor & Odds ratio & $95 \%$ CI & P-value \\
\hline Age & 0.98 & $0.94-1.02$ & 0.41 \\
Stature (5 cm interval) & 1.09 & $0.94-1.27$ & 0.25 \\
Body mass (5 kg interval) & 1.13 & $0.98-1.30$ & 0.083 \\
Total exposure (10 hour interval) & 1.02 & $1.00-1.04$ & 0.032 \\
Training / match exposure ratio & 1.00 & $0.99-1.02$ & 0.70 \\
Team home surface & & & \\
$\quad$ Natural grass & & & \\
$\quad$ Artificial turf & 1.00 & $0.70-1.67$ & 0.74 \\
Seasonal distribution & 1.08 & & \\
$\quad$ Autumn-spring season & & & \\
$\quad$ Spring-autum season & 1.00 & $0.65-1.43$ & 0.85 \\
Playing position & 0.96 & & \\
$\quad$ Goalkeeper & & & \\
$\quad$ Defender & 1.00 & $0.52-2.08$ & 0.91 \\
$\quad$ Midfielder & 1.04 & $0.46-1.75$ & 0.74 \\
$\quad$ Forward & 0.89 & $0.43-1.81$ & 0.74 \\
\hline
\end{tabular}

${ }^{1}$ Reference group 


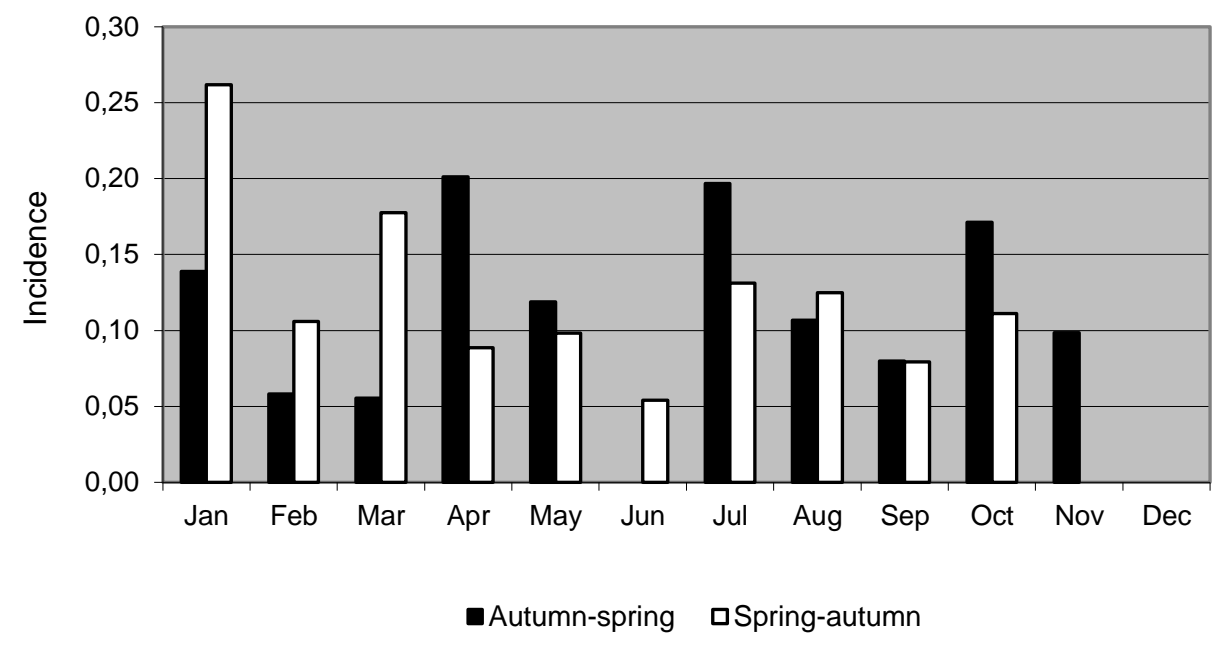

2 Figure 1. Incidence (injuries/1000 hours) of patellar tendinopathy over the season for teams following an 3 autumn-to-spring season (July-May, black bars) and a spring-to-autumn season (January-November, 4 white bars), respectively. 